\title{
Antibiotic Treatment of Suspected and Confirmed Neonatal Sepsis Within 28 Days of Birth: A Retrospective Analysis
}

\begin{abstract}
Jadon S. Wagstaff ${ }^{1}$, Robert J. Durrant ${ }^{2}$, Michael G. Newman ${ }^{3}$, Rachael Eason ${ }^{1,2}$, Robert M. Ward ${ }^{1}$, Catherine M. T. Sherwin ${ }^{4}$ and Elena Y. Enioutina ${ }^{1,2,5 *}$
\end{abstract}

\section{OPEN ACCESS}

Edited by:

Judith Ann Smith,

University of Texas Health Science

Center at Houston,

United States

Reviewed by:

Irja Lutsar,

University of Tartu, Estonia

Tracy Sandritter,

Children's Mercy Hospital,

United States

*Correspondence:

Elena Y. Enioutina

elena.enioutina@hsc.utah.edu

Specialty section: This article was submitted to

Obstetric and Pediatric

Pharmacology,

a section of the journal

Frontiers in Pharmacology

Received: 30 June 2019 Accepted: 17 September 2019

Published: 15 October 2019

Citation:

Wagstaff JS, Durrant RJ,

Newman MG, Eason R, Ward RM,

Sherwin CMT and Enioutina EY

(2019) Antibiotic Treatment of

Suspected and Confirmed Neonatal

Sepsis Within 28 Days of Birth: A

Retrospective Analysis.

Front. Pharmacol. 10:1191.

doi: 10.3389/fphar.2019.01191
Neonatal sepsis causes significant mortality and morbidity worldwide. Diagnosis is usually confirmed via blood culture results. Blood culture sepsis confirmation can take days and suffer from contamination and false negatives. Empiric therapy with antibiotics is common. This study aims to retrospectively describe and compare treatments of blood culture-confirmed and unconfirmed, but suspected, sepsis within the University of Utah Hospital system. Electronic health records were obtained from 1,248 neonates from January 1, 2006, to December 31, 2017. Sepsis was categorized into early-onset ( $\leq 3$ days of birth, EOS) and late-onset (>3 and $\leq 28$ days of birth, LOS) and categorized as culture-confirmed sepsis if a pathogen was cultured from the blood and unconfirmed if all blood cultures were negative with no potentially contaminated blood cultures. Of 1,010 neonates in the EOS cohort, 23 (2.3\%) were culture-confirmed, most with Escherichia coli (42\%). Treatment for unconfirmed EOS lasted an average of 6.1 days with primarily gentamicin and ampicillin while confirmed patients were treated for an average of 12.3 days with increased administration of cefotaxime. Of 311 neonates in the LOS cohort, 62 (20\%) were culture-confirmed, most culturing coagulase negative staphylococci (46\%). Treatment courses for unconfirmed LOS lasted an average of 7.8 days while confirmed patients were treated for an average of 11.4 days, these patients were primarily treated with vancomycin and gentamicin. The use of cefotaxime for unconfirmed EOS and LOS increased throughout the study period. Cefotaxime administration was associated with an increase in neonatal mortality, even when potential confounding factors were added to the logistic regression model (adjusted odds ratio 2.8, 95\% $\mathrm{Cl}[1.21,6.88], \mathrm{p}=0.02$ ). These results may not be generalized to all hospitals and the use of cefotaxime may be a surrogate for other factors. Given the low rate of blood culture positive diagnosis and the high exposure rate of empiric antibiotics, this patient population might benefit from improved diagnostics with reevaluation of antibiotic use guidelines.

Keywords: neonatal sepsis, antibiotic stewardship, rational prescribing, empiric therapy, newborns, bloodstream infections 


\section{INTRODUCTION}

Neonatal sepsis remains a significant cause of morbidity and mortality, particularly among preterm and low birth-weight neonates (Thaver and Zaidi, 2009; Shane et al., 2017). It is estimated that within 28 days of birth, $13 \%$ of neonatal mortality worldwide is caused by sepsis or meningitis (Liu et al., 2012). Neonatal sepsis is commonly divided into early-onset sepsis (EOS) and late-onset sepsis (LOS). EOS ( $\leq 3$ days of birth) is generally associated with organisms transferred to the neonate from the mother while LOS ( $>3$ days of birth) is usually associated with nosocomial or community acquired infections (Dong and Speer, 2015).

Diagnosing neonatal sepsis can be enigmatic-there is no consensus among clinicians or researchers. The current "gold standard" for diagnosis is a positive blood culture (Wynn et al., 2014). Blood culture-confirmed sepsis is only one facet of the disease; suspected sepsis in culture-negative cases is still considered clinical sepsis (Lukacs and Schrag, 2012; Wynn et al., 2014). Current recommendations call for empiric use of antibiotics before culture confirmation if sepsis is suspected, and possible continued antibiotic treatment if signs of sepsis persist when the culture is negative (Camacho-Gonzalez et al., 2013; Shane et al., 2017). Because of this approach, antibiotics are frequently administered in neonatal intensive care units (NICUs). In a study of 253,651 NICU neonatal records within the Pediatrix Medical Group database, the two most commonly administered drugs were ampicillin (69\%) and gentamicin (58\%); additionally, $18 \%$ of neonates were exposed to cefotaxime and $10 \%$ to vancomycin (Clark et al., 2006). With an increasing emphasis on antibiotic stewardship, it is essential to understand how neonatal sepsis is confirmed and treated, and to identify opportunities for reducing antibiotic use.

A retrospective analysis was conducted using data obtained from The University of Utah Health system, comprising four hospitals and 12 community clinics. Our objective was to thoroughly describe the blood culture confirmation and antibiotic treatment of neonatal sepsis within this hospital system. Antibiotics used to treat culture confirmed and unconfirmed sepsis are compared for both early and late onset sepsis over a 12 -year period.

\section{Methods}

Collection of medical records for analysis was approved by the University of Utah Institutional Review Board (IRB_00091312). Records were collected from neonates born and/or admitted to the University of Utah Hospitals within 28 days of birth between January 1, 2006, and December 31, 2017, with ICD9 billing code 771.81 or ICD10 code P36 (newborn sepsis). Neonates with major birth defects such as spina bifida, gastroschisis, congenital heart defects (excluding patent ductus arteriosus and patent foramen ovale), Down syndrome, encephalocele, and microcephaly or neonates born from mothers with a family history of congenital immune disorders (x-linked agammaglobulinemia, $\mathrm{x}$-linked neutropenia/myelodysplasia, common variable immunodeficiency, severe combined immunodeficiency, severe congenital neutropenia, Shwachman-Diamond syndrome, and Kostmann syndrome) were excluded from data collection.

We categorized these patients as suspected early-onset sepsis (EOS) when any blood culture was drawn and antibiotics were initiated $\leq 3$ days of birth and suspected late-onset sepsis (LOS) when any blood culture was drawn and antibiotics were administered $>3$ but $\leq 28$ days of birth and $\geq 7$ days after a positive blood culture associated with confirmed EOS. Neonates with suspected late or early-onset sepsis are not mutually exclusive, that is, a single neonate can be categorized as both suspected early and late-onset sepsis if it meets the criteria for both. Neonates suspected of having sepsis were further divided into two categories, confirmed sepsis and unconfirmed sepsis. These categories are mutually exclusive (i.e., a single neonate suspected of having LOS must be either confirmed or unconfirmed LOS).

Only blood cultures were used to confirm neonatal sepsis. Centers for Disease Control and Prevention (CDC) bloodstream infection criteria were adapted for use to confirm sepsis with some modifications (Horan et al., 2008). According to these guidelines, if there was a recognized pathogen in a blood culture, sepsis was confirmed. If a common skin contaminant was cultured, and the organism was cultured again in the following $48 \mathrm{~h}$, sepsis was also confirmed. If a common skin contaminant was cultured, and the organism was not cultured again in the following $48 \mathrm{~h}$, then these cultures were possibly contaminated and these patients were excluded from analysis as ambiguous cases. The following microorganisms were considered common skin contaminants: diphtheroids, Bacillus spp., Micrococcus spp., non-pathogenic Neisseria spp., gram-positive rods, coagulase negative staphylococci (CoNS), and non-pathogenic streptococcal species (e.g., Streptococcus viridans) (Hall and Lyman, 2006). We also analyzed cerebrospinal fluid (CSF) cultures.

Antibiotic exposure was measured by days of treatment (DOT) and length of treatment (LOT) (Ibrahim and Polk, 2014). DOT is measured by multiplying the number of doses by the dosing interval in days for each antibiotic administered. DOT is normalized to per 1,000 patient days (PD) which is the mean length of stay multiplied by 1,000 . LOT is measured by the number of calendar days a patient received any antibiotic. LOT is normalized to per admission and per treatment course. LOT/ course is equivalent to the mean number of days that neonates are treated for an episode of sepsis. Antibiotics administered topically were not included.

Treatment courses were calculated as continuous periods of antibiotic exposure with no gap in administration greater than or equal to two calendar days to accommodate for extended intervals of aminoglycoside dosing in extremely low birthweight newborns. In some cases, there was continuous administration of antibiotics from within three days of birth to well beyond three days after birth where there was an obvious switch from treatment of unconfirmed EOS to treatment of suspected LOS. For example, in one neonate, a negative culture was drawn on day one of life and ampicillin and gentamicin were administered as treatment for EOS. At day five of life, more cultures were drawn, ampicillin administration ceased, and vancomycin administration began as treatment for LOS. In these cases, we considered treatment courses ending for EOS and treatment courses beginning for 
LOS when there was a blood culture drawn after three days of birth, then a change in antibiotic regimen on or after the same calendar day.

Severity of illness and risk of mortality were measured using All Patient Refined Diagnosis Related Groups (APR-DRG). Univariate comparisons were evaluated using Student's $t$-test for continuous variables and chi-squared test or Fisher's exact test for categorical. Median values are reported with an interquartile range (IQR) in brackets and mean values are reported with standard deviation (SD) in parenthesis. Trends over time were analyzed using simple linear regression for continuous variables, logistic regression for dichotomous variables, or one-way ANOVA for severity of illness and risk of mortality. Statistical tests were considered significant when $\mathrm{p}<0.05$. All analyses were undertaken with $\mathrm{R}$ version 3.5 .3 ( $\mathrm{R}$ Core Team, 2019) in conjunction with the dplyr version 0.8.0 $\mathrm{R}$ package.

\section{RESULTS}

A total of 1,248 neonates met inclusion criteria for data collection, 1,010 met inclusion criteria for the EOS cohorts and 311 met inclusion criteria for the LOS cohorts (Figure 1). Patient demographics, mortality rate, length of stay, severity of illness, risk of mortality, birth weight, and gestational age for each cohort are compared in Table 1. Confirmed cases of LOS were more likely to include neonates with lower gestational age, lower birth weight, and higher severity of illness than neonates in unconfirmed cases of LOS ( $p<0.01)$.

Documentation about the source of the blood for the cultures was limited and unclear in many cases, but at least $10 \%$ were catheter-drawn, and at least $40 \%$ of those did not have an additional percutaneous culture within $\pm 48 \mathrm{~h}$. After the first positive culture in confirmed cases, the median interval between subsequent cultures was 0.98 days IQR $[0.62,1.48]$. Additionally, there were 389 CSF cultures drawn within 28 days of birth in this study cohort. Two neonates had positive CSF cultures, these are described below. Although most of the blood culture isolates were bacteria, there were six neonates that were infected with yeast. Three were infected with Candida lusitaniae, one with Candida dubliniensis, and the species of the remaining two could not be determined from the records.

Specific organisms associated with confirmed sepsis are outlined in Table 2. Blood cultures that were drawn within

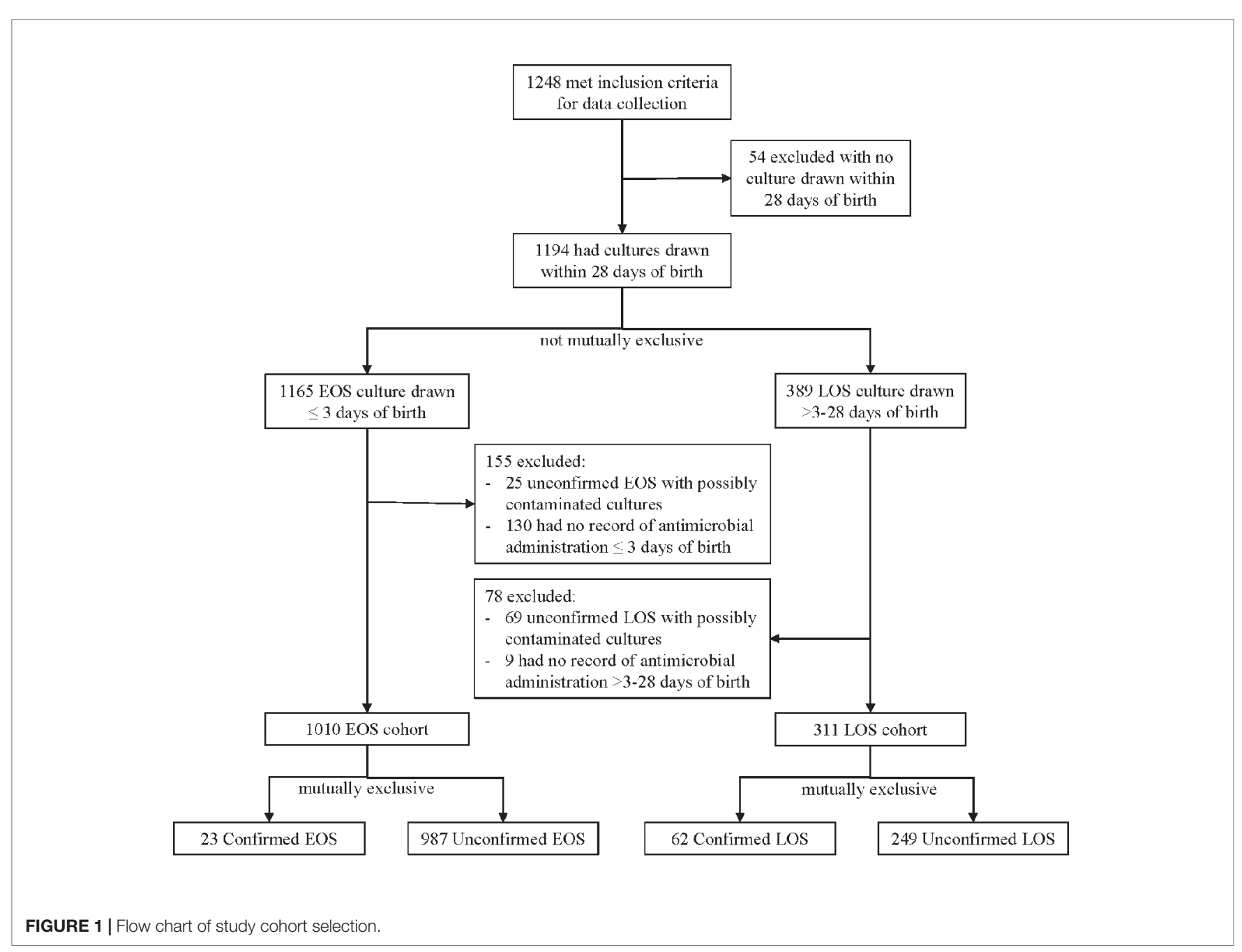


TABLE 1 | Cohort demographics.

\begin{tabular}{|c|c|c|c|c|c|c|}
\hline & \multicolumn{3}{|c|}{ Early-Onset } & \multicolumn{3}{|c|}{ Late-Onset } \\
\hline & Unconfirmed & Confirmed & P value & Unconfirmed & Confirmed & $P$ value \\
\hline Number of cases & 987 & 23 & & 249 & 62 & \\
\hline Mortality within 35 Days of Birth (\%) & $26(2.6)$ & $2(8.7)$ & 0.27 & $10(4.0)$ & $6(9.7)$ & 0.14 \\
\hline Length of Stay in Days (median [IQR]) & $23[8,57]$ & $41[15,57]$ & 0.50 & $58[34,101]$ & $69[23,101]$ & 0.72 \\
\hline \multicolumn{7}{|l|}{ Risk of Mortality (\%) } \\
\hline Extreme & $118(12.0)$ & $2(8.7)$ & 0.46 & $50(20.1)$ & $14(22.6)$ & 0.46 \\
\hline Major & $375(38.0)$ & $13(56.5)$ & & $134(53.8)$ & $36(58.1)$ & \\
\hline Moderate & $231(23.4)$ & $5(21.7)$ & & 38 (15.3) & $7(11.3)$ & \\
\hline Minor & $255(25.8)$ & $3(13.0)$ & & $24(9.6)$ & $3(4.8)$ & \\
\hline Missing & $8(0.8)$ & $0(0.0)$ & & $3(1.2)$ & $2(3.2)$ & \\
\hline \multicolumn{7}{|l|}{ Severity of Illness (\%) } \\
\hline Extreme & $319(32.3)$ & $11(47.8)$ & 0.42 & $134(53.8)$ & $45(72.6)$ & 0.04 \\
\hline Major & $342(34.7)$ & 8 (34.8) & & 88 (35.3) & $12(19.4)$ & \\
\hline Moderate & $223(22.6)$ & $2(8.7)$ & & $14(5.6)$ & $2(3.2)$ & \\
\hline Minor & $95(9.6)$ & $2(8.7)$ & & $10(4.0)$ & $1(1.6)$ & \\
\hline Missing & $8(0.8)$ & $0(0.0)$ & & $3(1.2)$ & $2(3.2)$ & \\
\hline \multicolumn{7}{|c|}{ Patients with other Positive Cultures within 28 Days of Birth } \\
\hline Lungs/Airway (\%) & $75(7.6)$ & $2(8.7)$ & 0.12 & $40(16.1)$ & $18(29.0)$ & 0.57 \\
\hline Urine (\%) & $34(3.4)$ & $0(0.0)$ & & $25(10.0)$ & $6(9.7)$ & \\
\hline Cerebrospinal Fluid (\%) & $3(0.3)$ & $1(4.3)$ & & $4(1.6)$ & $1(1.6)$ & \\
\hline \multicolumn{7}{|l|}{ Gestational Age (\%) } \\
\hline$>=37$ weeks (term) & $272(27.6)$ & $4(17.4)$ & 0.68 & $14(5.6)$ & $1(1.6)$ & $<0.01$ \\
\hline 32-37 weeks (preterm) & $281(28.5)$ & $7(30.4)$ & & $46(18.5)$ & $4(6.5)$ & \\
\hline 28-32 weeks (very preterm) & $212(21.5)$ & $5(21.7)$ & & $75(30.1)$ & $15(24.2)$ & \\
\hline$<28$ weeks (extreme preterm) & $222(22.5)$ & $7(30.4)$ & & $114(45.8)$ & $42(67.7)$ & \\
\hline \multicolumn{7}{|l|}{ Birth Weight (\%) } \\
\hline$>=2.5 \mathrm{~kg}$ (normal) & $332(33.6)$ & $4(17.4)$ & 0.18 & $19(7.6)$ & $2(3.2)$ & $<0.01$ \\
\hline $1.5-2.5 \mathrm{~kg}$ (low) & $275(27.9)$ & $6(26.1)$ & & 52 (20.9) & $6(9.7)$ & \\
\hline $1-1.5$ kg (very low) & $156(15.8)$ & $7(30.4)$ & & $61(24.5)$ & $9(14.5)$ & \\
\hline <1 kg (extremely low) & $224(22.7)$ & $6(26.1)$ & & $117(47.0)$ & 45 (72.6) & \\
\hline Male Gender (\%) & $535(54.2)$ & $14(60.9)$ & 0.81 & $118(47.4)$ & $33(53.2)$ & 0.50 \\
\hline \multicolumn{7}{|l|}{ Race (\%) } \\
\hline White or Caucasian & $528(53.5)$ & $11(47.8)$ & 0.68 & $140(56.2)$ & $35(56.5)$ & 0.58 \\
\hline Asian & $23(2.3)$ & $0(0.0)$ & & $3(1.2)$ & $1(1.6)$ & \\
\hline Black or African American & $26(2.6)$ & $0(0.0)$ & & $8(3.2)$ & $0(0.0)$ & \\
\hline Pacific Islander & $11(1.1)$ & 1 (4.3) & & $6(2.4)$ & $1(1.6)$ & \\
\hline American Indian & $12(1.2)$ & $0(0.0)$ & & $2(0.8)$ & $1(1.6)$ & \\
\hline Other & 102 (10.3) & $3(13.0)$ & & $28(11.2)$ & $4(6.5)$ & \\
\hline Unknown & 285 (28.9) & 8 (34.8) & & $62(24.9)$ & $20(32.3)$ & \\
\hline \multicolumn{7}{|l|}{ Ethnicity (\%) } \\
\hline Not Hispanic/Latino & $479(48.5)$ & $6(26.1)$ & 0.51 & $138(55.4)$ & $26(41.9)$ & 0.16 \\
\hline Hispanic/Latino & $173(17.5)$ & $9(39.1)$ & & $39(15.7)$ & $13(21.0)$ & \\
\hline Unknown & 335 (33.9) & $8(34.8)$ & & $72(28.9)$ & $23(37.1)$ & \\
\hline
\end{tabular}

28 days of birth in all sepsis cohorts numbered 2,483; within these, $223(8.98 \%)$ contained organisms considered as pathogens and $118(4.75 \%)$ cultures were identified as possibly contaminated. Of the cultures that were possibly contaminated, 21 (17.8\%) did not have a second blood culture draw within $\pm 48 \mathrm{~h}$. Of the organisms identified as possible contaminants, only coagulase-negative staphylococci (CoNS) was also identified as a potential pathogen. Out of 226 cultures that were positive for CoNS, 122 (54.0\%) were categorized as pathogenic.

\section{Early-Onset Sepsis}

There were 1,010 neonates within the EOS cohorts, $23(2.3 \%)$ were classified as confirmed cases of EOS and $987(97.7 \%)$ as unconfirmed (Figure 1). There were 24 culture isolates identified that were associated with EOS (Table 2); 1 of 23 (4.3\%) neonates with confirmed EOS had more than one type of non-contaminant organism (Escherichia coli with yeast). The most common organisms identified as EOS pathogens were Escherichia coli (43.5\% of neonates) and group B streptococcus (17.4\%). One confirmed EOS patient infected with Escherichia coli had both blood and CSF cultures positive for Escherichia coli.

The ratio of culture confirmed to unconfirmed EOS cases remained consistent over time $(p=0.59)$. Because of the low number of confirmed EOS cases and the consistent occurrence, trends over time were analyzed for the combined confirmed and unconfirmed EOS cohorts. There was no statistically significant change in severity of illness and risk 
TABLE 2 | Pathogens identified in culture-confirmed sepsis.

\begin{tabular}{lcc}
\hline Organism & $\begin{array}{c}\text { Early-onset sepsis* } \\
\mathbf{( \% )} \\
\mathbf{n = 2 4}\end{array}$ & $\begin{array}{c}\text { Late Onset sepsis* } \\
\mathbf{( \% )}\end{array}$ \\
\hline Gram-positive bacteria & & $\mathbf{n}$ \\
CoNS & $1(4.2)$ & $31(45.6)$ \\
Enterococcus spp. & $1(4.2)$ & $7(10.3)$ \\
Staphylococcus aureus & & $7(10.3)$ \\
Group B Streptococcus & $4(16.7)$ & $\mathrm{N} / \mathrm{D}$ \\
Listeria monocytogenes & $1(4.2)$ & $\mathrm{N} / \mathrm{D}$ \\
Streptococcus pneumoniae & $1(4.2)$ & $\mathrm{N} / \mathrm{D}$ \\
Gram-negative bacteria & & \\
Escherichia coli & $10(41.7)$ & $9(13.2)$ \\
Klebsiella pneumoniae & $2(8.3)$ & $3(4.4)$ \\
Enterobacter spp. & $\mathrm{N} / \mathrm{D}$ & $2(2.9)$ \\
Serratia marcescens & $\mathrm{N} / \mathrm{D}$ & $2(2.9)$ \\
Haemophilus influenzae & $2(8.3)$ & $\mathrm{N} / \mathrm{D}$ \\
Acinetobacter sp. & $\mathrm{N} / \mathrm{D}$ & $1(1.5)$ \\
Citrobacter sedlakii & $\mathrm{N} / \mathrm{D}$ & $1(1.5)$ \\
Neisseria meningitidis & $1(4.2)$ & $\mathrm{N} / \mathrm{D}$ \\
Yeast & $1(4.2)$ & $5(7.4)$ \\
& &
\end{tabular}

${ }^{*}$ Number of unique patient-organism combinations. (A patient may have cultured more than one organism.); N/D, not detected.

of mortality over time. The average gestational age and birth weight for EOS neonates increased over the study period from 30.4 to 34.5 weeks and 1.50 to $2.45 \mathrm{~kg}$ respectively $\left(R^{2}=0.05\right.$, $\mathrm{p}<0.01$ for both). The average length of stay for EOS neonates decreased from of 45.9 days at the beginning of the study period to 32.8 days at the end $\left(\mathrm{R}^{2}=0.05, \mathrm{p}<0.01\right)$. Logistic regression showed that there was a statistically significant increase in the rate of mortality over time for EOS neonates (odds ratio 1.27/year, 95\%CI [1.10, 1.49], $\mathrm{p}<0.01$ ).

Confirmed EOS neonates had more exposure to antibiotics than unconfirmed EOS neonates by all metrics in Table 3 $(\mathrm{p}<0.01)$. Unconfirmed EOS neonates were mostly prescribed ampicillin and gentamicin (Figure 2B); $77.2 \%$ of the LOT for courses starting within 3 days of birth in these patients included both ampicillin and gentamicin in combination. Confirmed EOS neonates were treated with only one antibiotic more often than unconfirmed EOS neonates ( $46.3 \%$ and $9.3 \%$ of LOT respectively, Figure 2A). For confirmed EOS neonates, $65.3 \%$ of the LOT for courses started within 3 days of birth included cefotaxime either alone or in combination with other antibiotics, $35.1 \%$ consisted of treatment with only cefotaxime. Cefotaxime was most common antibiotic for confirmed EOS neonates (Figure 2B). Fifteen of the confirmed EOS neonates received cefotaxime starting within
3 days of birth for a mean of 11.8 days LOT, SD (6.0). Gram negative microorganisms were cultured in 13 of the 15 confirmed EOS patients treated with cefotaxime and two of the patients died during the course of treatment.

There was no statistically significant changes in EOS course LOT over the study period for both unconfirmed and confirmed EOS ( $p=0.45,0.24$ respectively). There was no significant change in the rates of administration of any antibiotic for confirmed EOS. Unconfirmed EOS courses saw a reduction in the rate of gentamicin administration and an increase in the rate of cefotaxime administration where very few courses included cefotaxime in at the beginning of the study period, to nearly $30 \%$ of courses including cefotaxime at the end of 2017 (Table 4).

\section{Late-Onset Sepsis}

Of 311 neonates within the LOS cohorts, 62 (19.9\%) were classified as confirmed cases of LOS, $249(80.1 \%)$ as unconfirmed. A total of 68 culture isolates were identified as pathogens associated with LOS (Table 2); 4 of 62 (6.5\%) confirmed LOS neonates were positive for multiple organisms (fist patient-CoNS, Klebseilla pneumonia, Entercoccus faecalis, and Candida lusitaniae; second-Staphylococcus aureus and Enterococcus faecalis; thirdCoNS and Acinetobacter sp.; fourth-Staphylococcus aureus and Staphylococcus epidermidis). The most common pathogens causing LOS were CoNS (50.0\% of neonates) followed by Escherichia coli (14.5\%). One of the unconfirmed LOS neonates had one positive CSF culture within $>3-28$ days of birth which isolated Candida dubliniensis, no blood cultures were drawn within \pm 4 days of this CSF culture.

Similar to confirmation rates in EOS, the ratio of culture confirmed to unconfirmed LOS cases also remained consistent over time $(\mathrm{p}=0.80)$. There was no statistically significant change in severity of illness, risk of mortality, gestational age, birth weight, and length of stay over time when unconfirmed and confirmed LOS were analyzed both combined and separated. Mortality rates within 35 days of birth increased over time for unconfirmed LOS (odds ratio 1.37/year, 95\%CI [1.10, 1.81], p = 0.01 ) and confirmed LOS (odds ratio 1.42 /year, 95\%CI [1.06, 2.17], $\mathrm{p}=0.04)$.

The most common antibiotics used in neonates with unconfirmed and confirmed LOS werevancomycin and gentamicin (Figures 2C-D). Similar to neonates with EOS, confirmed LOS neonates had more exposure to antibiotics than unconfirmed LOS by all metrics (Figure 2C, Table 3, p $<0.01$ ). For courses starting within $>3-28$ days of birth, rifampin was more frequently

TABLE 3 | Measurements of total antibiotic exposure per cohort.

\begin{tabular}{|c|c|c|c|c|}
\hline \multirow[t]{2}{*}{ Metric } & \multicolumn{2}{|c|}{ Early-Onset Sepsis* } & \multicolumn{2}{|c|}{ Late-Onset Sepsis* } \\
\hline & Suspected & Confirmed & Suspected & Confirmed \\
\hline DOT/1000 PD (SD) & $513(477)$ & $582(334)$ & $472(314)$ & $634(385)$ \\
\hline LOT/Admission (SD) & $10.9(10.0)$ & $15.9(7.0)$ & $17.9(11.5)$ & $23.5(14.9)$ \\
\hline LOT/Course (starting $\leq 3$ days of birth) (SD) & $6.1(3.4)$ & $12.3(6.2)$ & N/A & $\mathrm{N} / \mathrm{A}$ \\
\hline LOT/Course (starting >3-28 days of birth) (SD) & N/A & N/A & $7.8(5.7)$ & $11.4(9.4)$ \\
\hline
\end{tabular}

${ }^{\star}$ The differences between suspected and confirmed sepsis were statistically significant for all metrics $(p<0.01)$. 
A - EOS

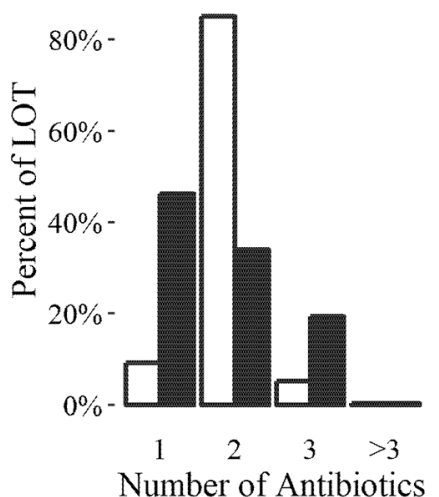

C - LOS

$80 \%-$

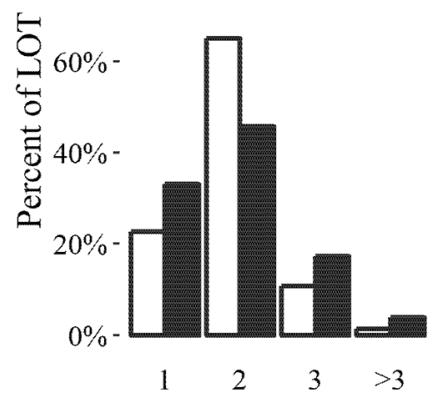

Number of Antibiotics
B - EOS

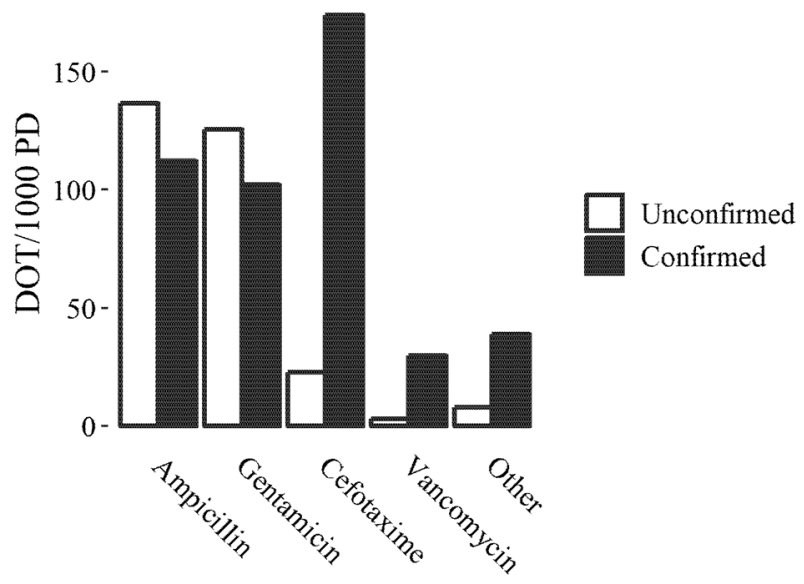

D - LOS

150 -

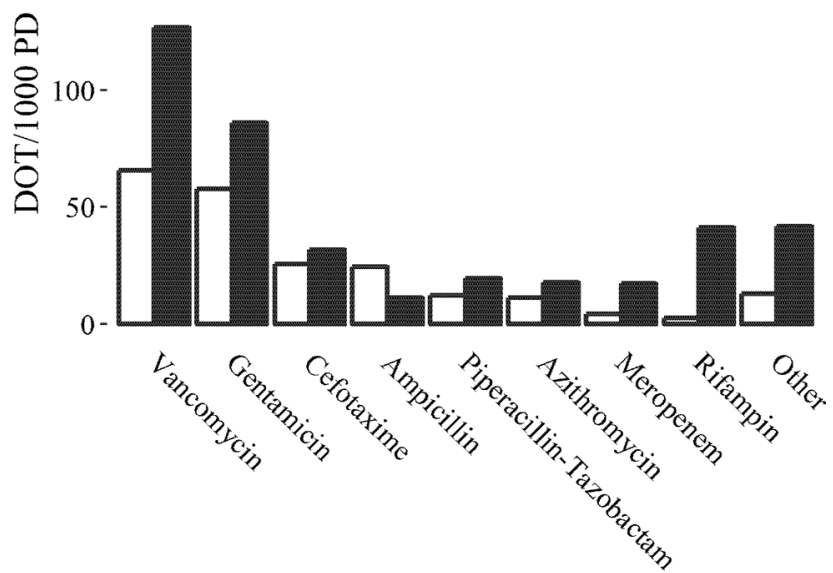

FIGURE 2 | Antibiotic use for treatment of neonatal sepsis. (A and C) number of antibiotics administered per calendar day as a percentage of LOT per cohort. (B and D)-amount of each antibiotic administered as DOT/1,000 PD per cohort. (A and B) figures for early onset sepsis (EOS): only courses started within 3 days of birth are included. (C and D) figures for late onset sepsis (LOS): only courses started within $>3-28$ of birth were included.

TABLE 4 | Antibiotics with significant changes in administration rates over time. Results of logistic regression modeling with antibiotic administration rates at the beginning and end of the time period (1/1/2006 - 12/31/2017).

\begin{tabular}{|c|c|c|c|c|}
\hline \multirow[t]{2}{*}{ Antibiotic } & \multirow[t]{2}{*}{ Odds ratio/year $(95 \% \mathrm{Cl})$} & \multirow[t]{2}{*}{$\mathbf{P}$} & \multicolumn{2}{|c|}{ Courses with Administration } \\
\hline & & & Beginning & End \\
\hline \multicolumn{5}{|c|}{ Unconfirmed Early Onset Sepsis } \\
\hline Cefotaxime & $1.25(1.17,1.34)$ & $<0.01$ & $2.8 \%$ & $29.7 \%$ \\
\hline Gentamicin & $0.69(0.57,0.80)$ & $<0.01$ & $99.8 \%$ & $87.2 \%$ \\
\hline \multicolumn{5}{|c|}{ Unconfirmed Late Onset Sepsis } \\
\hline Ampicillin & $1.10(1.02,1.19)$ & 0.02 & $28.9 \%$ & $55.6 \%$ \\
\hline Cefotaxime & $1.24(1.14,1.37)$ & $<0.01$ & $8.4 \%$ & $55.8 \%$ \\
\hline Gentamicin & $0.78(0.70,0.85)$ & $<0.01$ & $92.4 \%$ & $36.3 \%$ \\
\hline Piperacillin-Tazobactam & $1.24(1.12,1.39)$ & $<0.01$ & $4.6 \%$ & $39.8 \%$ \\
\hline Vancomycin & $0.83(0.75,0.89)$ & $<0.01$ & $86.8 \%$ & $38.7 \%$ \\
\hline \multicolumn{5}{|c|}{ Confirmed Late Onset Sepsis } \\
\hline Gentamicin & $0.78(0.65,0.91)$ & $<0.01$ & $94.0 \%$ & $42.8 \%$ \\
\hline Rifampin & $0.79(0.65,0.93)$ & $<0.01$ & $58.3 \%$ & $7.1 \%$ \\
\hline
\end{tabular}


administered to confirmed LOS neonates than unconfirmed (41.2 and 2.6 DOT/1000 PD respectively, Figure 2D). In all 22 cases of confirmed LOS treated with rifampin, the neonates were infected with CoNS and treatment was always combined with vancomycin. The mean time difference between the first positive culture draw and the start of rifampin treatment was 4.0 days SD (1.8). In 17 neonates $(77 \%)$, there were no positive cultures after initiation of rifampin treatment, for the remaining neonates the mean time to the last positive culture after treatment was started was 1.85 days SD (1.92). One patient died after 2.5 days of treatment with rifampin; this patient had a positive culture drawn just prior to death. There was no statistically significant changes in LOS course LOT over the study period for both unconfirmed and confirmed LOS ( $\mathrm{p}=0.93,0.57$ respectively). There was a reduction in the rate of rifampin administration in cases of confirmed LOS, a reduction in vancomycin administration for unconfirmed LOS, and a reduction in gentamicin administration over time in both cohorts (Table 4). Courses for unconfirmed LOS saw an increase in the inclusion of ampicillin, piperacillin-tazobactam, and cefotaxime (Table 4).

\section{Neonatal Mortality}

As described, patient mortality had statistically significant increasing trend over the study period for unconfirmed EOS and LOS and confirmed LOS. For the combined EOS and LOS cohorts, a logistic regression model estimated a mortality rate within 35 days of birth of $0.4 \%$ at the beginning of the study period to $8.5 \%$ of patients at the end (odds ratio $1.30 /$ year, $95 \% \mathrm{CI}$ $[1.13,1.52], \mathrm{p}<0.01)$. Additionally, the use of cefotaxime was included in treatment courses for unconfirmed EOS and LOS at an increasing rate during the study period (Table 4). For combined EOS and LOS neonates, the inclusion of cefotaxime within 28 days of birth went from $5.6 \%$ of courses to $37.6 \%$ of courses (odds ratio 1.21, 95\%CI $[1.16,1.27], \mathrm{p}<0.01$ ) over the course of the study period.

When mortality was modeled as a function of whether neonates had exposure to cefotaxime within 28 days of birth, cefotaxime exposure had a significant impact on mortality (odds ratio 7.92, 95\% CI [3.76, 17.79], $\mathrm{p}<0.01$ ). This association remained when gestational age, birth weight, hospital admission date-time, blood culture confirmation of sepsis, risk of mortality, and severity of illness were added to the logistic regression model as potential confounders (adjusted odds ratio 2.8, 95\%CI [1.21, 6.88], $\mathrm{p}=0.02$ ).

\section{DISCUSSION}

\section{Confirmation of Neonatal Sepsis}

Establishing blood culture-confirmed neonatal sepsis is complicated by the presence of false negative culture results and culture contamination. False negative cultures are commonly attributed to low volume of blood or low pathogen concentrations, which is an inherent problem in the mostly low body weight newborns with neonatal sepsis (Kellogg et al., 2000; Dien Bard and McElvania TeKippe, 2016). Additionally, it is believed by some that exposure to intrapartum antibiotics when treating chorioamnionitis or other maternal illnesses can reduce sensitivity of blood cultures (Peralta-Carcelen et al., 1996). To overcome these limitations, it is recommended that $\geq$ $\geq 1 \mathrm{ml}$ of blood is collected for culturing (Polin, 2012; Dien Bard and McElvania TeKippe, 2016). Using $1 \mathrm{ml}$ of blood for a blood culture has been demonstrated to provide $98 \%$ sensitivity even when pathogen concentrations are low (4 colony forming units per ml) (Schelonka et al., 1996). The volume of cultured blood was not available in this study, therefore the effect of blood volume on false negative results in this hospital system are difficult to infer.

Even though a blood culture may provide a sensitive diagnostic tool, the results may take several days. In the past, attempts have been made to detect true cases of sepsis earlier than a blood culture, (Philip and Hewitt, 1980) but there is no consensus on how to incorporate laboratory tests in diagnosing neonatal sepsis (Wynn et al., 2014). New biomarkers such as bacterial surface antigens and emerging technologies such as genetic sequencing based tools may be used more frequently in neonatal sepsis diagnostics in the future (Chauhan et al., 2017; Iroh Tam and Bendel, 2017). Automated multiplex PCR systems can provide pathogen specific identification of common microorganisms including many organisms found in neonates such as E.coli and S. aureus in as little as an hour, (Salimnia et al., 2016) but it is unknown whether all pathogens are adequately detectable.

Regardless of the method used to detect microorganisms in a blood sample, sample contamination can mislead diagnosis. To complicate the issue, when a central venous catheter is used to draw blood, positive cultures may be due to catheter colonization (Hall and Lyman, 2006). Some organisms, such as Escherichia coli, Staphylococcus aureus, and Streptococcus pneumoniae, are almost always associated with a true infection, while others are commonly contaminants, such as CoNS and Streptococcus viridans (Hall and Lyman, 2006). When a common contaminant is cultured, misdiagnosis is reduced by obtaining and comparing multiple cultures; and when blood is drawn from a catheter, a second sample should be drawn percutaneously (Schifman et al., 1998; Hall and Lyman, 2006). CoNS are the most common blood culture contaminants, (Hall and Lyman, 2006) but they are also a prominent causative agent of LOS (Dong and Speer, 2015). In this study, approximately $54 \%$ of cultures positive for CoNS were identified as true positives, $46 \%$ were identified as potentially contaminated. Additionally, at least $15 \%$ of CoNS-positive cultures were from catheter-drawn blood samples. Distinguishing between contaminants and pathogens is imperative in these cases. CoNS cultures cannot simply be completely excluded from, or completely included in, culture-confirmed cases.

Clinical signs and laboratory results indicating sepsis beyond blood cultures are commonly used to classify confirmed cases of sepsis (Bizzarro et al., 2005; Haque, 2005; Wynn et al., 2014; Zea-Vera and Ochoa, 2015). Incorporating any of these methods into confirming sepsis retrospectively has shortcomings; in particular, data are sometimes sparse. It is important to realize that when laboratory results or clinical signs remain abnormal but blood cultures are negative, sepsis may still be suspected and the use of antibiotics may be prolonged. To allow comparisons with other studies and since blood cultures are the 
gold standard for the diagnosis of neonatal sepsis, the criteria for retrospectively confirming neonatal sepsis via a modified version of CDC guidelines (Horan et al., 2008) within this study used only culture results.

\section{Treatments for Neonatal Sepsis}

Of neonates treated for EOS in the University of Utah Hospital system, only $2.3 \%$ had blood culture confirmation and there was a high rate of empiric use of ampicillin and gentamicin. This type of empiric treatment is common practice in neonatal intensive care units (NICUs) within the United States. Oliver et al. reported that within the Pediatric Health Information System, $92.5 \%$ of neonates in a NICU that were administered antibiotics within three days of birth received ampicillin and/ or gentamicin on the first day of treatment (Oliver et al., 2017). Cefotaxime appeared to be a preferred choice in therapy for confirmed EOS with gram-negative infections for the University of Utah Hospital system.

Empiric treatment for LOS seems to vary more than empiric treatment for EOS, but within the University of Utah Hospital system, vancomycin and/or gentamicin were the most common treatments. In a 2002 survey of 278 neonatology clinicians at 35 hospitals with NICUs, at least $60 \%$ prescribed vancomycin as part of empiric therapy for unconfirmed LOS, commonly combined with an aminoglycoside (Rubin et al., 2002). Prudent use of vancomycin has been advised for decades due to the evolution of vancomycin resistant strains of pathogens (The Hospital Infection Control Practices Advisory Committee, 1995). There is some evidence that empiric vancomycin does not improve short-term mortality or length of stay when neonates are infected with CoNS (Ericson et al., 2015). Within the University of Utah Hospital system, there are signs of improvement in the antibiotic stewardship of vancomycin where the DOT/1,000 PD for vancomycin use was $37.3 \%$ lower in unconfirmed compared to confirmed cases of LOS with a significant reduction in empiric use over time (Table 4). Rifampin was often combined with vancomycin to treat persistent CoNS infections within the University of Utah Hospital systemthere is some limited evidence that this strategy is effective (Acar et al., 1983; Shama et al., 2002; van der Lugt et al., 2010; RodriguezGuerineau et al., 2013).

Neonates with unconfirmed sepsis within the University of Utah Hospital system were treated for a median of 7 days LOT IQR (Clark et al., 2006; Liu et al., 2012), which is similar to, or longer than, treatment lengths for unconfirmed sepsis in similar studies within the United States and Europe (Patel et al., 2009; Cantey et al., 2015; Fjalstad et al., 2016). Antibiotic administration may be prolonged even when cultures are negative if blood cell counts, $C$ reactive protein levels, or other diagnostic tests remain abnormal. Continued administration of antibiotics when cultures are negative appears to be a common practice. This is in spite of evidence that prolonged empirical antibiotic administration within neonates $(\geq 5$ days) is associated with adverse outcomes such as necrotizing enterocolitis, prolonged hospital stay, and death (Kuppala et al., 2011; Ting et al., 2016). Recent studies on the incubation time for blood cultures in cases of neonatal sepsis suggest that antibiotic treatment can be ceased after $48 \mathrm{~h}$ if cultures remain negative (Jardine et al., 2006; Durrani et al., 2019) Cantey et al. demonstrated that when empiric neonatal antibiotic therapy was set to automatically discontinue after $48 \mathrm{~h}$ and continued therapy was limited to 5 days when cultures were negative, there was no difference in safety outcomes compared to prior practices where antibiotics were regularly administered $\geq 6$ days when cultures were negative (Cantey et al., 2016).

Since the gestational age, birth weight, severity of illness, risk of mortality, and the ratio of culture confirmed to unconfirmed cases of neonatal sepsis remained fairly consistent throughout the study period, it was unexpected that the neonatal sepsis mortality rate within the University of Utah Hospital system increased over the study period. In 2006, Clark et al. showed that the concurrent use of ampicillin and cefotaxime within 3 days of birth rather than ampicillin with gentamicin had an increased risk of mortality (Clark et al., 2006). Logistic modeling showed that the use of cefotaxime within 28 days of birth may be a cause of the increased neonatal sepsis mortality within the University of Utah hospital system, even after adjusting for potential confounders. This conclusion is limited by the retrospective nature of this study: cefotaxime administration may be a surrogate for some other unknown factor. Further analysis of the causes of death and the motivations for prescribing cefotaxime may reveal other factors that may be responsible for the increased mortality rate.

One potential limitation of this study is that antibiotic therapy may have been prolonged for neonates with infections other than sepsis such as pneumonia or meningitis. In our patient cohorts, there were no neonates that had both negative blood cultures and positive CSF culture which would suggest neonatal meningitis. When neonates with any other positive cultures were removed from the unconfirmed cohorts (see Table 1), the LOT/course for courses starting $\leq 3$ days of birth reduced from 6.1 to 6.0 for unconfirmed EOS and the LOT/course for courses starting $>3-28$ days of birth reduced from 7.8 to 7.5 for unconfirmed LOS. Both of these differences were insignificant ( $p>0.05$ ); therefore, we believe that the main findings of this study are still supported. This study has other limitations. Comorbidities and mother's characteristics were not accounted for and may affect suspicion of sepsis and choices in antibiotic treatments. The results of this study cannot be generalized to other hospitals since epidemiology and practices are likely to differ from region to region.

\section{TWELVE-YEAR OVERVIEW}

Over the past 12 years, the level of empirical treatment remained high. The major limitations to reduce empirical treatment are highly variable clinical presentation of sepsis in neonates and low levels of confirmed sepsis. The development or improvement of diagnostic tests capable of accurately confirming sepsis within 1-2 $\mathrm{h}$ after blood draw is a key factor that will reduce empirical treatment of neonatal sepsis. One of the possible risk factors for the development of sepsis in neonates is an underdeveloped immune defences (Gervassi and Horton, 2014). Recently, it has been proposed that immune responses in neonates are suppressed by afterbirth residual myeloid derived suppressor 
cells (MDSCs) (Gervassi and Horton, 2014). MDSCs are a heterogeneous population of immature myeloid cells capable of expanding following pathologic conditions such as infections and cancer. MDSCs have been detected in the blood of septic patients (Cuenca et al., 2011). The ontogenetic destiny of MDSCs is to suppress the fetal immune response against the mother. MDSCs possess the remarkable ability to suppress $\mathrm{T}, \mathrm{B}$, and NK cell functions and by this mean impair protective immune responses to infectious agents (Rieber et al., 2013; Gervassi et al., 2014). Presence of these cells in cord blood can potentially serve as early biomarker of neonatal sepsis. If MDSCs indeed suppress anti-microbial immunity of neonates, the immune system can be restored and sepsis development prevented by blocking MDSCs activity or by differentiation of MDSCs into mature myeloid cells lacking suppressive activities. This approach may reduce the rate of neonatal sepsis, empirical use of antibiotics, and increase survival of preterm babies.

\section{CONCLUSIONS}

In conclusion, the large proportion of unconfirmed to confirmed neonatal sepsis combined with a high empiric antibiotic administration rate and high level of exposure indicates an opportunity to reduce unnecessary antibiotic use in the neonatal population. This may be accomplished by implementing new and improved methods for more sensitive and timely diagnosis

\section{REFERENCES}

Acar, J. F., Goldstein, F. W., and Duval, J. (1983). Use of rifampin for the treatment of serious staphylococcal and gram-negative bacillary infections. Rev. Infect. Dis. 5 (Supplement_3), S502-S506. doi: 10.1093/clinids/5.Supplement_3. S502

Bizzarro, M. J., Raskind, C., Baltimore, R. S., and Gallagher, P. G. (2005). Seventyfive years of neonatal sepsis at yale: 1928-2003. Pediatrics 116 (3), 595-602. doi: 10.1542/peds.2005-0552

Camacho-Gonzalez, A., Spearman, P. W., and Stoll, B. J. (2013). Neonatal infectious diseases: evaluation of neonatal sepsis. Pediatr. Clin. North. Am. 60 (2), 367-389. doi: 10.1016/j.pcl.2012.12.003

Cantey, J. B., Wozniak, P. S., and Sánchez, P. J. (2015). Prospective surveillance of antibiotic use in the neonatal intensive care unit: results from the SCOUT study. Pediatr. Infect. Dis. J. 34 (3), 267-272. doi: 10.1097/INF.0000000000000542

Cantey, J. B., Wozniak, P. S., Pruszynski, J. E., and Sánchez, P. J. (2016). Reducing unnecessary antibiotic use in the neonatal intensive care unit (SCOUT): a prospective interrupted time-series study. Lancet Infect. Dis. 16 (10), 11781184. doi: 10.1016/S1473-3099(16)30205-5

Chauhan, N., Tiwari, S., and Jain, U. (2017). Potential biomarkers for effective screening of neonatal sepsis infections: an overview. Microb. Pathog. 107, 234242. doi: 10.1016/j.micpath.2017.03.042

Clark, R. H., Bloom, B. T., Spitzer, A. R., and Gerstmann, D. R. (2006). Reported medication use in the neonatal intensive care unit: data from a large national data set. Pediatrics 117 (6), 1979-1987. doi: 10.1542/peds.2005-1707

Clark, R. H., Bloom, B. T., Spitzer, A. R., and Gerstmann, D. R. (2006). Empiric use of ampicillin and cefotaxime, compared with ampicillin and gentamicin, for neonates at risk for sepsis is associated with an increased risk of neonatal death. Pediatrics 117 (1), 67-74. doi: 10.1542/peds.2005-0179

Cuenca, A. G., Delano, M. J., Kelly-Scumpia, K. M., Moreno, C., Scumpia, P. O., Laface, D. M., et al. (2011). A paradoxical role for myeloid-derived suppressor cells in sepsis and trauma. Mol. Med. 17 (3-4), 281-292. doi: 10.2119/ molmed.2010.00178 of neonatal sepsis and reevaluating guidelines for appropriate antibiotic use.

\section{DATA AVAILABILITY STATEMENT}

The dataset for this manuscript is not publicly available because it contains personal information on human subjects. The dataset cannot be completely de-identified and shared without compromising the privacy of research participants.

\section{ETHICS STATEMENT}

The studies involving human participants were reviewed and approved by University of Utah Institutional Review Board (IRB_00091312). Written informed consent from the participants' legal guardian/next of kin was not required to participate in this study in accordance with the national legislation and the institutional requirements.

\section{AUTHOR CONTRIBUTIONS}

JW, MN and RD undertook the analysis, EE and CS designed and conceptualized the study, EE, CS, RE and RW were involved in study design and data analysis. All authors were engaged in the writing and review of the manuscript drafts and final submission.

Dien Bard, J., and McElvania TeKippe, E. (2016). Diagnosis of bloodstream infections in children. J. Clin. Microbiol. 54 (6), 1418-1424. doi: 10.1128/ JCM.02919-15

Dong, Y., and Speer, C. P. (2015). Late-onset neonatal sepsis: recent developments. Arch. Dis. Child Fetal Neonatal. 100 (3), F257-F263. doi: 10.1136/ archdischild-2014-306213

Durrani, N., Rochow, N., Alghamdi, J., Pelc, A., Fusch, C., and Dutta, S. (2019). Minimum duration of antibiotic treatment based on blood culture in ruled out neonatal sepsis. Pediatr. Infect. Dis. J. 38 (5), 528-532. doi: 10.1097/ INF.0000000000002182

Ericson, J. E., Thaden, J., Cross, H. R., Clark, R. H., Fowler, V. G. Jr., Benjamin D. K. Jr., et al. (2015). No survival benefit with empirical vancomycin therapy for coagulase-negative staphylococcal bloodstream infections in infants. Pediatr. Infect. Dis. J. 34 (4), 371-375. doi: 10.1097/INF.0000000000000573

Fjalstad, J. W., Stensvold, H. J., Bergseng, H., Simonsen, G. S., Salvesen, B., Rønnestad, A. E., et al. (2016). Early-onset sepsis and antibiotic exposure in term infants: a nationwide population-based study in Norway. Pediatr. Infect. Dis. J. 35 (1), 1-6. doi: 10.1097/INF.0000000000000906

Gervassi, A. L., and Horton, H. (2014). Is infant immunity actively suppressed or immature? Virology (Auckl) 2014 (5), 1-9. doi: 10.4137/VRT.S12248

Gervassi, A., Lejarcegui, N., Dross, S., Jacobson, A., Itaya, G., Kidzeru, E., et al. (2014). Myeloid derived suppressor cells are present at high frequency in neonates and suppress in vitro T cell responses. PLoS One 9 (9), e107816. doi: 10.1371/journal.pone.0107816

Hall, K. K., and Lyman, J. A. (2006). Updated review of blood culture contamination. Clin. Microbiol. Rev. 19 (4), 788-802. doi: 10.1128/CMR.00062-05

Haque, K. N. (2005). Definitions of bloodstream infection in the newborn. Pediatr. Crit. Care Med. 6 (Supplement), S45-S49. doi: 10.1097/01. PCC.0000161946.73305.0A

Horan, T. C., Andrus, M., and Dudeck, M. A. (2008). CDC/NHSN surveillance definition of health care-associated infection and criteria for specific types of infections in the acute care setting. Am. J. Infect. Control. 36 (5), 309-332. doi: 10.1016/j.ajic.2008.03.002 
Ibrahim, O. M., and Polk, R. E. (2014). Antimicrobial use metrics and benchmarking to improve stewardship outcomes. Infect. Dis. Clin. N. Am. 28 (2), 195-214. doi: 10.1016/j.idc.2014.01.006

Iroh Tam, P.-Y., and Bendel, C. M. (2017). Diagnostics for neonatal sepsis: current approaches and future directions. Pediatr. Res. 82 (4), 574-583. doi: 10.1038/ pr.2017.134

Jardine, L., Davies, M. W., and Faoagali, J. (2006). Incubation time required for neonatal blood cultures to become positive. J. Paediatr. Child Health 42 (12), 797-802. doi: 10.1111/j.1440-1754.2006.00980.x

Kellogg, J. A., Manzella, J. P., and Bankert, D. A. (2000). Frequency of low-level bacteremia in children from birth to fifteen years of age. J. Clin. Microbiol. 38 (6), 2181-2185.

Kuppala, V. S., Meinzen-Derr, J., Morrow, A. L., and Schibler, K. R. (2011). Prolonged initial empirical antibiotic treatment is associated with adverse autcomes in premature infants. J. Pediatr. 159 (5), 720-725. doi: 10.1016/j. jpeds.2011.05.033

Liu, L., Johnson, H. L., Cousens, S., Perin, J., Scott, S., Lawn, J. E., et al. (2012). Global, regional, and national causes of child mortality: an updated systematic analysis for 2010 with time trends since 2000. Lancet 379 (9832), 2151-2161. doi: 10.1016/S0140-6736(12)60560-1

Lukacs, S. L., and Schrag, S. J. (2012). Clinical sepsis in neonates and young infants, United States, 1988-2006. J. Pediatr. 160 (6), 960-965.e1. doi: 10.1016/j. jpeds.2011.12.023

Oliver, E. A., Reagan, P. B., Slaughter, J. L., Buhimschi, C. S., and Buhimschi, I. A. (2017). Patterns of empiric antibiotic administration for presumed early-onset neonatal sepsis in neonatal intensive care units in the United States. Amer J. Perinatol. 34 (07), 640-647. doi: 10.1055/s-0036-1596055

Patel, S. J., Oshodi, A., Prasad, P., Delamora, P., Larson, E., Zaoutis, T., et al. (2009). Antibiotic use in neonatal intensive care units and adherence with Centers for Disease Control and Prevention 12 Step Campaign to Prevent Antimicrobial Resistance. Pediatr. Infect. Dis. J. 28 (12), 1047-1051. doi: 10.1097/INF.0b013e3181b12484

Peralta-Carcelen, M., Fargason, Jr. CA, Cliver, S. P., Cutter, G. R., Gigante, J., and Goldenberg, R. L. (1996). Impact of maternal group B streptococcal screening on pediatric management in full-term newborns. Arch. Pediatr. Adolesc. Med. 150 (8), 802-808. doi: 10.1001/archpedi.1996.02170330028005

Philip, A. G. S., and Hewitt, J. R. (1980). Early diagnosis of neonatal sepsis. Pediatrics 65 (5), 1036-1041.

Polin, R. A. (2012). Committee on Fetus and Newborn. Management of neonates with suspected or proven early-onset bacterial sepsis. Pediatrics 129 (5), 10061015. doi: 10.1542/peds.2012-0541

R Core Team (2019). R: A Language and Environment for Statistical Computing. Austria: Vienna. https://www.R-project.org/.

Rieber, N., Gille, C., Kostlin, N., Schafer, I., Spring, B., Ost, M., et al. (2013). Neutrophilic myeloid-derived suppressor cells in cord blood modulate innate and adaptive immune responses. Clin. Exp. Immunol. 174 (1), 45-52. doi: $10.1111 /$ cei. 12143

Rodriguez-Guerineau, L., Salvia-Roigés, M. D., León-Lozano, M., RodríguezMiguélez, J. M., and Figueras-Aloy, J. (2013). Combination of vancomycin and rifampicin for the treatment of persistent coagulase-negative staphylococcal bacteremia in preterm neonates. Eur. J. Pediatr. 172 (5), 693-697. doi: 10.1007/ s00431-012-1927-x

Rubin, L. G., Sánchez, P. J., Siegel, J., Levine, G., Saiman, L., and Jarvis, W. R. (2002). Evaluation and treatment of neonates with suspected late-onset sepsis: a survey of neonatologists' practices. Pediatrics 110 (4), e42-e42. doi: 10.1542/peds.110.4.e42
Salimnia, H., Fairfax, M. R., Lephart, P. R., Schreckenberger, P., DesJarlais, S. M., Johnson, J. K., et al. (2016). Evaluation of the FilmArray blood culture identification panel: results of a multicenter controlled trial. J. Clin. Microbiol. 54 (3), 687-698. doi: 10.1128/JCM.01679-15

Schelonka, R. L., Chai, M. K., Yoder, B. A., Hensley, D., Brockett, R. M., and Ascher, D. P. (1996). Volume of blood required to detect common neonatal pathogens. J. Pediatr. 129 (2), 275-278. doi: 10.1016/ S0022-3476(96)70254-8

Schifman, R. B., Strand, C. L., Meier, F. A., and Howanitz, P. J. (1998). Blood culture contamination: a College of American Pathologists Q-Probes study involving 640 institutions and 497134 specimens from adult patients. Arch. Pathol. Lab. Med. 122 (3), 216-221.

Shama, A., Patole, S. K., and Whitehall, J. S. (2002). Intravenous rifampicin in neonates with persistent staphylococcal bacteraemia. Acta Paediatr. 91 (6), 670-673. doi: 10.1111/j.1651-2227.2002.tb03300.x

Shane, A. L., Sánchez, P. J., and Stoll, B. J. (2017). Neonatal sepsis. Lancet 390 (10104), 1770-1780. doi: 10.1016/S0140-6736(17)31002-4

Thaver, D., and Zaidi, A. K. M. (2009). Burden of neonatal infections in developing countries: a review of evidence from community-cased studies. Pediatr. Infect. Dis. J. 28 (Supplement), S3-S9. doi: 10.1097/ INF.0b013e3181958755

The Hospital Infection Control Practices Advisory Committee (1995). Recommendations for preventing the spread of vancomycin resistance: recommendations of the Hospital Infection Control Practices Advisory Committee (HICPAC). Am. J. Infect. Control. 23 (2), 87-94. doi: 10.1016/0196-6553(95)90104-3

Ting, J. Y., Synnes, A., Roberts, A., Deshpandey, A., Dow, K., Yoon, E. W., et al. (2016). Association between antibiotic use and neonatal mortality and morbidities in very low-birth-weight infants without culture-proven sepsis or necrotizing enterocolitis. JAMA Pediatr. 170 (12), 1181-1187. doi: 10.1001/ jamapediatrics.2016.2132

van der Lugt, N. M., Steggerda, S. J., and Walther, F. J. (2010). Use of rifampin in persistent coagulase negative staphylococcal bacteremia in neonates. $B M C$ Pediatr. doi: 10.1186/1471-2431-10-84

Wynn, J. L., Wong, H. R., Shanley, T. P., Bizzarro, M. J., Saiman, L., and Polin, R. A. (2014). Time for a neonatal-specific consensus definition for sepsis. Pediatr. Crit. Care Med. 15 (6), 523-528. doi: 10.1097/PCC.0000000000000157

Zea-Vera, A., and Ochoa, T. J. (2015). Challenges in the diagnosis and management of neonatal sepsis. J. Trop. Pediatr. 61 (1), 1-13. doi: 10.1093/ tropej/fmu079

Conflict of Interest: CS is the Specialty Chief Editor of the section Obstetric and Pediatric Pharmacology within Frontiers in Pharmacology.

The remaining authors declare that the research was conducted in the absence of any commercial or financial relationships that could be construed as a potential conflict of interest.

Copyright (c) 2019 Wagstaff, Durrant, Newman, Eason, Ward, Sherwin and Enioutina. This is an open-access article distributed under the terms of the Creative Commons Attribution License (CC BY). The use, distribution or reproduction in other forums is permitted, provided the original author(s) and the copyright owner(s) are credited and that the original publication in this journal is cited, in accordance with accepted academic practice. No use, distribution or reproduction is permitted which does not comply with these terms. 